\title{
Can Self-Reported Personal Audio System Volume Predict Actual Listening Levels in Young Adults?
}

DOI: $10.3766 /$ jaaa.17104

Peter Torre III*

Mark B. Reed $\dagger$

\begin{abstract}
Background: Most young adults report using personal audio systems (PAS) with earphones as part of their daily activities. PAS exposure is intermittent and research examining the levels these young adults are listening to is increasing. On average, preferred listening levels are below what would be considered at risk in an occupational setting.
\end{abstract}

Purpose: The purpose of this study was to evaluate how two questions predicted preferred listening level in young adults with normal hearing; specifically, whether these young adults could identify if they listen at a high level or not.

Study Sample: One hundred and sixty young adults (111 women, 49 men) with normal hearing completed a questionnaire that had questions about PAS listening habits and then had preferred listening level assessed using a probe microphone system while listening to 1 hour of music through earphones.

Data Collection and Analysis: Otoscopy, tympanometry, and pure-tone thresholds were completed in a randomly determined test ear. As part of the Risk Factors Survey, two closed-set questions were completed. First, "For a typical day, what is the most common volume used during this day?" with the following response options "Low," "Medium/Comfortable," "Loud," or "Very Loud." And second, "Do you listen to your personal music system at a volume where you. .." with the following response options "Easily hear people," "Have a little trouble hearing people," "Have a lot of trouble hearing people," or "Cannot hear people." Using a probe microphone, chosen listening level (A-weighted, diffuse-field correction and a conversion to free-field equivalent $\left[L_{D F e q}\right]$ ) was calculated over 1 hour while the participant listened to music with earphones. Sensitivity and specificity were determined to see how well young adults could identify themselves as listening at a high level $(>85 \mathrm{dBA})$ or not. Linear regression analyses were performed to determine the amount of variance explained by the two survey questions as predictors of measured $L_{\mathrm{DFeq}}$.

Results: Almost half of the participants reported a longest single use of a PAS as $<1$ hour daily and more than half reported listening at a medium/comfortable volume and had a little trouble hearing people. Mean $L_{\text {DFeq }}$ was $72.5 \mathrm{dBA}$, with young adult men having a significantly higher mean $L_{\text {DFeq }}(76.5 \mathrm{dBA})$ compared with young adult women (70.8 dBA). Sensitivity was $88.9 \%$ and specificity was $70.6 \%$ for the question asking about volume on a typical day. For the question asking about being able to hear other people while listening to music sensitivity was $83.3 \%$ and specificity was $82.5 \%$. Two variables, listening volume on a typical day and sex, accounted for $28.4 \%$ of the variability associated with $L_{D F e q}$; the answer to the question asking about being able to hear others and sex accounted for $22.8 \%$ of the variability associated with $L_{\text {DFeq. }}$

Conclusions: About $11 \%$ of young adults in the present study listen to a PAS with earphones at a high level $(>85 \mathrm{dBA})$ while in a quiet background. The participants who do report listening at a high level, however, do well at self-reporting this risk behavior in survey questions.

Key Words: listening levels, risk factors, young adults

Abbreviations: $F F E=$ free-field equivalent; $L_{\text {Aeq }}=A$-weighted equivalent continuous noise level; $L_{\mathrm{DFeq}}=$ diffuse-field correction, free-field equivalent level; $L_{\mathrm{PASe}}=$ daily personal audio system use exposure; $L_{\mathrm{pmse}}=$ daily personal music system exposure; $\mathrm{PAS}=$ personal audio system; $\mathrm{SD}=$ standard deviation

*School of Speech, Language, and Hearing Sciences, San Diego State University, San Diego, CA; †School of Social Work, San Diego State University, San Diego, CA

Corresponding author: Peter Torre III, School of Speech, Language, and Hearing Sciences, San Diego State University, San Diego, CA 92182-1518; Email: ptorre@mail.sdsu.edu 


\section{INTRODUCTION}

$\mathrm{A}$ high percentage (i.e., 90-95\%) of college-aged young adults report owning a personal audio system (PAS) with earphones (Torre, 2008; Danhauer et al, 2009). However, within the last 5-10 yr, the PAS devices have transitioned from ones such as an iPod, Walkman, or a compact disc player to the iPhone or Android device, which allow for more music storage and longer listening opportunities. PAS use with earphones is a recreational noise exposure that is an intermittent exposure, different from occupational noise exposure that can be impulse in nature or more likely, a constant, higher level exposure. Occupational noise exposure, and the evaluation of risk for noise-induced hearing loss is federally monitored by the Occupational Safety and Health Administration (OSHA, 2007) and the National Institute for Occupational Safety and Health (NIOSH, 1998). These standards, however, were based on an 8-hour workday and a permissible time-weighted average level (i.e., 90dBA time-weighted average for Occupational Safety and Health Administration). Industrial types of noise will vary from setting to setting, can have either a narrower frequency response or a more broad frequency, but the sound source can be at a distance from the worker. Music, on the other hand, will also have a broader frequency response, and, when the user is wearing earphones, will be delivered in close proximity to the tympanic membrane. Because exposure to music through earphones varies both by number of hours within the day and number of days within the week, calculations of risk can be made but by using an 8-hour equivalent of a recreational noise exposure day.

Because of the ubiquitous aspect of PASs with earphones, especially on college/university campuses (Torre, 2008; Danhauer et al, 2009; Hoover and Krishnamurti, 2010), there are more researchers objectively evaluating the preferred listening levels of young adults using probe microphone measures (Hodgetts et al, 2007; Torre, 2008; Worthington et al, 2009; Torre and Grace, 2014; Park et al, 2017). Preferred listening levels can range, on average, as low as $58 \mathrm{dBA}$ (Torre and Grace, 2014) but more likely at levels of $~ 70-80 \mathrm{dBA}$ (Hodgetts et al, 2007; Torre, 2008; Worthington et al, 2009; Park et al, 2017), with young adult men having higher preferred listening levels compared with women. However, the sex difference for volume/preferred listening level is not consistent in the literature because some researchers have not reported a difference in women and men (Epstein et al, 2010; Hoover and Krishnamurti, 2010).

In one of the few studies to evaluate reported PAS use and preferred listening level, Worthington et al (2009) developed an equation, $L_{\text {pmse }}=L_{\text {Aeq }}+10 \log (T / 8)$, where $L_{\text {pmse }}$ is the level of daily personal music exposure, $L_{\text {Aeq }}$ is the A-weighted equivalent continuous noise level, and $T$ is the reported use time per day, in hours. This equation combined the reported number of hours of PAS use with the level measured from the ear canal using a probe microphone. In this study, 30 normal-hearing young adults ( 18 women and 12 men; mean age $=22 \mathrm{yr}$, standard deviation $[\mathrm{SD}]=3.4 \mathrm{yr}$ ) participated and none of the 30 young adults listened at a hazardous level, in quiet. In fact, even when listening in the presence of a background noise and accounting for diffuse-field equivalency, only one participant was determined to be listening at a level $>85 \mathrm{dBA}$.

Because of the cost of probe microphones and the procedure involved in measuring the preferred listening level, researchers have also used questionnaires to assess preferred listening volume (Torre, 2008; Danhauer et al, 2009; Torre et al, 2013). Danhauer et al (2009) used both an online questionnaire as well as a paperand-pencil questionnaire in more than 600 college students whereas Torre (2008) completed intervieweradministered questionnaires in just more than 1,000 university students. Just more than $50 \%$ of respondents in both studies reported a typical listening volume of medium, but Torre (2008) found that more than $40 \%$ reported either loud or very loud listening volumes compared with 34\% reported by Danhauer et al (2009). Torre (2008) also found that men were significantly more like likely to report very loud as their preferred listening volume compared with women. What is not presently known is how subjective assessments of preferred listening volume (i.e., medium, loud, or very loud) compare with objective measures of chosen listening level using a probe microphone system.

Researchers have evaluated whether certain questions (i.e., Do you feel you have a hearing loss?) can be a strong predictor of audiometrically defined hearing loss (Clark et al, 1991; Voeks et al, 1993; Nondahl et al, 1998). Voeks et al (1993) used the question, "Do you have trouble hearing?" with nursing home residents and reported 69\% sensitivity (defined as residents who correctly identified themselves as having a hearing loss). In 60- to 85-yr-old women, Clark et al (1991) used a similar question, "Would you say that you have any difficulty hearing?" and found $51 \%$ sensitivity and $88 \%$ specificity (women who correctly identified themselves as having normal hearing). Last, Nondahl et al (1998) used the question, "Do you feel you have a hearing loss?" to evaluate how 48 to $92 \mathrm{yr}$ olds identify themselves as having a hearing loss. That screening question had $71 \%$ for both sensitivity and specificity. From an epidemiologic perspective, the goal of any screening measure, or survey question, is to maximize both sensitivity and specificity, but if researchers have to make a choice, then that depends on what is being identified. If, for example, the goal is to identify the presence of a disease or risk behavior, then maximizing sensitivity would be 
best. If the choice is to identify those without the disease or risk behavior, then maximizing specificity would be optimal.

As mentioned previously, no screening question has been evaluated to predict whether young adults are engaging in risky listening behavior with PASs. As a result, the purpose of the present study was to evaluate how two questions predicted chosen listening level in young adults with normal hearing. The main objective was to calculate sensitivity and specificity and determine if one or two questions could be used in young adults to examine whether they listen to a PAS with earphones at potentially high levels. The implication of this is that the question(s) could be then used in a clinical case history form to assist in counseling for noise-induced hearing loss.

\section{METHODS}

\section{Participants}

From Table 1, 160 San Diego State University (SDSU) undergraduate students were recruited from Exercise and Nutritional Sciences, Public Health, and Social Work course (i.e., non-Speech, Language, and Hearing Sciences courses). There were 111 women (mean age $=20.9 \mathrm{yr}, \mathrm{SD}=2.6 \mathrm{yr}$ ) and 49 men (mean age $=20.9 \mathrm{yr}, \mathrm{SD}=3.3 \mathrm{yr}$ ) who volunteered for the study and received extra credit in their course for their participation. More than one-quarter $(28.1 \%)$ of participants reported their ethnicity as Hispanic or Latino and $113(70.6 \%)$ participants reported their ethnicity as Not Hispanic or Latino, 2(1.3\%) declined to state ethnicity. Participants also had the opportunity to provide racial background data, and the data for the specific racial categories are shown in Table 1.

Table 1. Sex, Ethnicity, and Race Characteristics of the Study Participants

\begin{tabular}{lrr}
\hline & \multicolumn{2}{c}{ Participants $(\mathrm{n}=160)$} \\
\cline { 2 - 3 } & $\mathrm{n}$ & Percent \\
\hline Women & 111 & 69.4 \\
Men & 49 & 30.6 \\
Ethnicity ( $\mathrm{n}=160)$ & & \\
$\quad$ Hispanic or Latino & 45 & 28.1 \\
$\quad$ Not Hispanic or Latino & 113 & 70.6 \\
$\quad$ Decline to state & 2 & 1.3 \\
Race (n = 160) & & \\
$\quad$ American Indian/Alaska Native & 1 & 0.6 \\
$\quad$ Asian & 23 & 14.4 \\
$\quad$ Native Hawaiian/Pacific Islander & 5 & 3.1 \\
$\quad$ Black/African American & 12 & 7.5 \\
$\quad$ White & 98 & 61.3 \\
$\quad$ Decline to state & 21 & 13.1 \\
\hline
\end{tabular}

\section{Procedures}

These data are a portion of the data that were included in the Risk Factors for Hearing Loss in Young Adults Study approved by the SDSU Institutional Review Board. Other data from this study (i.e., pure-tone thresholds and distortion product otoacoustic emissions) will be reported elsewhere. Once informed consent was obtained, research assistants administered the Risk Factors Survey. A section of this survey included demographic questions of sex, age, ethnicity, race, and questions specific to PAS use. If the participant answered No to the question, "Do you listen to a personal music system using earphones?" then that part of the survey was completed. If Yes, then additional questions regarding type of earphone used, typical duration of listening, longest single use during the day, most common volume used, and if they noticed any problems (e.g., ringing and hearing loss) after using a personal music system were completed. Two of the closed-set survey questions of interest in this study were "For a typical day, what is the most common volume used during this day?" "Low" (coded as 1 for statistical analyses), "Medium/Comfortable" (2), "Loud" (3), or "Very Loud" (4); and "Do you listen to your personal music system at a volume where you..." "Easily hear people" (coded as 1 for statistical analyses), "Have a little trouble hearing people" (2), "Have a lot of trouble hearing people" (3), or "Cannot hear people" (4). All participants had normal hearing $(\leq 25 \mathrm{~dB})$ from 250 through $8000 \mathrm{~Hz}$, including 3000 and $6000 \mathrm{~Hz}$ and normal middle ear function measured with tympanometry (i.e., type A tympanogram).

With the participant seated in a double-walled soundtreated room (Industrial Acoustics Company, Inc., North Aurora, IL), chosen listening level data for this quiet setting were collected using an ER 7C Probe Microphone Series B (Etymotic Research, Inc., Elk Grove Village, IL) system set to a 0-dB gain connected to Electroacoustics Toolbox software (Version 3.8.3; Faber Acoustical, LLC) on an iMac (Apple, Inc., Cupertino, CA) computer. A probe microphone was placed in the ear canal at an insertion depth of approximately $28 \mathrm{~mm}$ from the end of the probe to the intertragal notch. This depth is common for probe microphone measures in adults because the proximity to the tympanic membrane will most likely reduce the effect of standing waves within the ear canal (Dirks and Kincaid, 1987).

Within the Electroacoustics Toolbox, the Sound Level Meter and Octave Band Analyzer functions were used. The Sound Level Meter function was used to measure the $L_{\text {Aeq }}$ whereas the Octave Band Analyzer was used to collect 1/3 octave data of the music participants listened to. Before chosen listening level data were obtained, the probe microphone system was calibrated; the ER 7C 
system has a built-in $1 \mathrm{kHz}, 94.0-\mathrm{dB}$ SPL calibration tone along with a probe microphone calibration cavity. To ensure the microphone was calibrated with the Electroacoustics Toolbox software, the probe microphone was placed in the cavity, the tone was presented, and the software, specifically the Sound Level Meter function, was required to display 94.0-dB SPL. Once calibration was completed, the probe microphone was secured in the test ear canal in two ways: a small piece of medical tape on the ear lobe, and the participant's preferred earphones. The participant then listened to 1 hour of continuous music, and they were allowed to change music and the level as they desired. When 1 hour was finished, the average $L_{\text {Aeq }}$ and $1 / 3$ octave band data were exported from the Electroacoustic Toolbox software for off-line analyses.

\section{Statistical Analyses}

All questionnaire data were analyzed using a $\chi^{2}$ approach (SAS, Version 9.4; SAS Institute, Cary, NC) to evaluate these categorical data for any differences between women and men across the questions. Probe microphone $1 / 3$ octave band data between 200 and $8000 \mathrm{~Hz}$ for each participant were put into an Excel spreadsheet to calculate diffuse-field equivalent level $\left(L_{\mathrm{DFeq}}\right)$ for a closed canal and converted to free-field equivalent (FFE). This same Excel spreadsheet was used by Worthington et al (2009) so $L_{\mathrm{DFeq}}$ could be interpreted using the National Institute for Occupational Safety and Health standard (i.e., $85 \mathrm{dBA}$ ). This spreadsheet, however, only allows for a conversion for earbud earphones. Furthermore, daily PAS use exposure $\left(L_{\mathrm{PASe}}\right)$ was calculated as $L_{\mathrm{PASe}}=L_{\mathrm{DFeq}}+10 \log (T / 8)$, where $T$ is the reported use time per day, in hours, by the participant during the survey. This formula is adapted from Worthington et al (2009), where the current approach substitutes $L_{\mathrm{DFeq}}$ for $L_{\mathrm{Aeq}}$. Analyses involving $L_{\mathrm{DFeq}}$ and $L_{\text {PASe }}$ data were completed using PROC GLM (generalized linear model) in SAS (Version 9.4; SAS Institute). Independent variables for these analyses included sex, age, ethnicity, and earphone type.

Two separate approaches were used to evaluate how well survey questions could determine if an individual would objectively listen to music using earphones at a hazardous level. First, $2 \times 2$ contingency tables were generated to calculate sensitivity and specificity of the two survey questions, separately, as they relate to exposure risk. Participant responses to the question asking about typical volume of PAS were categorized into two groups: Non-Loud (comprised of low and medium/comfortable responses) and Loud (comprised of loud and very loud). Participants were also categorized into two groups based upon their responses to the question asking about whether they can hear others when listening to their PAS. Specifically, the category Can
Hear was comprised of participants who reported they could easily hear people or have a little trouble hearing people. The category Cannot Hear included combined responses of having a lot of trouble hearing people and cannot hear people. Chosen listening level (i.e., $L_{\mathrm{DFeq}}$ ) was stratified as $\leq 85 \mathrm{dBA}$ or $>85 \mathrm{dBA}$. Sensitivity was defined as those individuals who either reported Loud as a common daily volume or Cannot Hear people while listening to music and listened at $>85 \mathrm{dBA}$ and specificity was defined as those individuals who either reported Non-Loud as a common daily volume or Can Hear people while listening to music and listened at $\leq 85 \mathrm{dBA}$. Linear regression analysis (SAS, Version 9.4; SAS Institute) was used to determine the amount of variance explained by the two survey questions as predictors of measured $L_{\mathrm{DFeq}}$.

\section{RESULTS}

$\mathrm{F}$ ive participants (four women and one man) reported not using a PAS with earphones; these participants were only included in $L_{\mathrm{DFeq}}$ analyses. Most of the participants who reported listening to music with earphones $(n=155)$ listened between 1 and 7 hours/week, at a medium/comfortable volume, and had a little trouble hearing other people while using earphones (Table 2 ). Also in Table $2,17.4 \%(\mathrm{n}=27)$ noticed ringing in their ears after listening to music through earphones whereas $6.5 \%(\mathrm{n}=10)$ felt that they had a hearing loss. Those who reported ringing in their ears did not report a significantly higher amount of loud or very loud volume $\left(\chi^{2}(1)=1.09, p>0.05\right)$ or higher rates of $>85 \mathrm{dBA}$ $\left(\chi^{2}(1)=0.68, p>0.05\right)$. However, with such a low percentage of participants reporting ringing in their ears, these results should be interpreted cautiously. Men reported a significantly higher rate of loud or very loud as the common volume used compared with women $\left(\chi^{2}(3)=\right.$ $8.64, p<0.05)$. More than $75 \%$ of participants $(\mathrm{n}=118)$ reported total daily listening time between $<1$ and 3 hours (Figure 1), and this figure shows the distribution of responses by women and men, in percentages. This difference between women and men was not statistically significant $\left(\chi^{2}(9)=14.10, p>0.05\right)$. Figure 2 shows the reported longest single daily use for men and women and once again, more than $75 \%$ of participants $(\mathrm{n}=$ 119) reported between $<1$ and 1 hour, but the difference in reported use between women and men was not statistically significant $\left(\chi^{2}(4)=4.19, p>0.05\right)$.

The distribution for $L_{\mathrm{DFeq}}$ stratified for percentages of women and men is shown in Figure 3. The distribution for men is shifted slightly higher than the distribution for women. Mean $L_{\mathrm{DFeq}}$ for all participants was 72.5 $\mathrm{dBA}(\mathrm{SD}=11.0 \mathrm{~dB}$; minimum $=49.4 \mathrm{dBA}$; maximum $=$ $94.9 \mathrm{dBA}$ ); after adjusting for age, earphone type used, and ethnicity, women had a significantly lower mean $L_{\mathrm{DFeq}}($ mean $=70.8 \mathrm{dBA} ; \mathrm{SD}=10.6 \mathrm{dBA})$ than men 
Table 2. Participant Responses $(n=155)$ to the HearingRelated Questions of the Risk Factors for Hearing Loss in Young Adults Study Survey

\begin{tabular}{|c|c|}
\hline Question & $\begin{array}{c}\text { Response } \\
\mathrm{n}(\%)\end{array}$ \\
\hline \multicolumn{2}{|l|}{$\begin{array}{l}\text { How would you describe your weekly } \\
\text { personal music system listening use? }\end{array}$} \\
\hline Low $(<1$ hour/week $)$ & $23(14.8)$ \\
\hline Medium (1-7 hours/week) & $92(59.4)$ \\
\hline Heavy ( $>7$ hours/week) & $40(25.8)$ \\
\hline \multicolumn{2}{|l|}{$\begin{array}{l}\text { For a typical day, what is the longest } \\
\text { single use during this day (in hours)? }\end{array}$} \\
\hline$<1$ & $74(47.7)$ \\
\hline 1 & $45(29.0)$ \\
\hline 2 & $25(16.1)$ \\
\hline 3 & $10(6.5)$ \\
\hline 4 & $1(0.7)$ \\
\hline \multicolumn{2}{|c|}{ 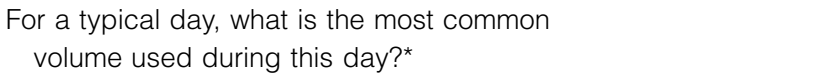 } \\
\hline Low & $1(0.6)$ \\
\hline Medium/comfortable & $97(63.0)$ \\
\hline Loud & $48(31.2)$ \\
\hline Very Loud & $8(5.2)$ \\
\hline \multicolumn{2}{|c|}{$\begin{array}{l}\text { Do you listen to your personal music system } \\
\text { at a volume where you }\end{array}$} \\
\hline easily hear people & $16(10.3)$ \\
\hline have a little trouble hearing people & $100(64.5)$ \\
\hline have a lot of trouble hearing people & $22(14.2)$ \\
\hline cannot hear people & $17(11.0)$ \\
\hline \multicolumn{2}{|c|}{$\begin{array}{l}\text { Have you noticed any problem with your hearing } \\
\text { after using a personal music system? }\end{array}$} \\
\hline No & $135(87.1)$ \\
\hline Yes & $6(3.9)$ \\
\hline Not sure & $14(9.0)$ \\
\hline \multicolumn{2}{|c|}{$\begin{array}{l}\text { Have you noticed any ringing in your ears after } \\
\text { using a personal music system? }\end{array}$} \\
\hline No & $122(78.7)$ \\
\hline Yes & $27(17.4)$ \\
\hline Not sure & $6(3.9)$ \\
\hline \multicolumn{2}{|c|}{$\begin{array}{l}\text { Did you know that prolonged use of a personal music } \\
\text { system at high volume can lead to hearing loss? }\end{array}$} \\
\hline No & $15(9.7)$ \\
\hline Yes & $137(88.4)$ \\
\hline Not sure & $3(1.9)$ \\
\hline \multicolumn{2}{|l|}{ Do you feel that you have a hearing loss? } \\
\hline No & $127(81.9)$ \\
\hline Yes & $10(6.5)$ \\
\hline Not sure & $18(11.6)$ \\
\hline
\end{tabular}

Note: Five participants reported not being a personal music system user and were not asked any listening use questions.

${ }^{*}$ One participant reported Not Sure to this question.

$($ mean $=76.5 \mathrm{dBA} ; \mathrm{SD}=10.8 \mathrm{dBA})\left[F_{(1,153)}=9.32, p<\right.$ 0.05]. But 18 participants $(11.3 \%)$ did listen at a level $>85$ $\mathrm{dBA} ; 9$ women (8.1\%) and 9 men (18.4\%) listened at this level. The main effect for earphone type was not statistically significant $\left[F_{(2,153)}=0.42, p>0.05\right]$; the adjusted means for earphones were similar (Apple earbuds $[\mathrm{n}=$
92], mean $=71.6 \mathrm{dBA}[\mathrm{SD}=11.1 \mathrm{dBA}]$; insert-type earphones $[\mathrm{n}=47]$, mean $=73.9 \mathrm{dBA}[\mathrm{SD}=9.6 \mathrm{dBA}]$; and over-the-ear earphones $[\mathrm{n}=21]$, mean $=73.7 \mathrm{dBA}$ $[\mathrm{SD}=13.1 \mathrm{dBA}])$. The association between earphone type and reported trouble hearing other people was analyzed, and there was no significant effect for earphone type $\left(\chi^{2}\right.$ $(2)=1.26, p>0.05)$. $L_{\text {PASe }}$ was also calculated for those 155 participants who reported using a PAS with earphones. The distribution for $L_{\mathrm{PASe}}$ stratified for percentages of women and men is shown in Figure 4. Similar to Figure 3, the distribution for men is shifted slightly higher than the distribution for women, where there are substantially more women in the $<60 \mathrm{dBA}$ category. Overall mean $L_{\mathrm{PASe}}$ was $66.3 \mathrm{dBA}(\mathrm{SD}=11.9 \mathrm{dBA})$. After adjusting for age, earphone type, and ethnicity, women had a significantly lower mean $L_{\mathrm{PASe}}$ of $64.0 \mathrm{dBA}$ $(\mathrm{SD}=11.7 \mathrm{dBA})$ than men who had a mean $L_{\mathrm{PASe}}$ of $71.3 \mathrm{dBA}(\mathrm{SD}=10.9 \mathrm{dBA})\left[F_{(1,148)}=13.38, p<\right.$ $0.05]$. This result is based on men having a significantly higher mean chosen listening level and having a higher percentage of longer reported daily listening times. In fact, of the nine participants with an $L_{\mathrm{PASe}}>85 \mathrm{dBA}$, six were men.

Sensitivity for the question asking about the most common volume used during this day was $88.9 \%$ (16 of 18 ) whereas specificity for this question was $70.6 \%$ (96 of 136). In other words, this means that $29.4 \%$ reported loud or very loud volume, yet did not listen at a high listening level (defined as false alarms), whereas only $11.1 \%$ ( 2 of 18) reported Non-Loud responses to this question, but listened at a high level (defined as misses). For the question asking participants to report whether listening to their PAS affects hearing others, sensitivity was $83.3 \%$ ( 15 of 18 ) and specificity was $82.5 \%$ (113 of 137). This means that $17.5 \%$ reported that they had a lot of trouble hearing others or could not hear others, yet did not listen at a high listening level (defined as false alarms), whereas $16,7 \%$ reported that they could hear people while using their PAS, but did listen at a high listening level (defined as misses). Both of these survey items had high sensitivity and specificity values although the second question had stronger combined sensitivity and specificity values.

Result of the linear regression analysis showed that self-reported typical listening volume accounted for $25.9 \%$ of the variability associated with preferred listening level, $L_{\mathrm{DFeq}}(Y=50.2+9.3[$ Loud]; see "Methods" section for coding of the responses to this question). Given the significant differences in chosen listening level as a function of Sex (coded as 0 for women and 1 for men), Sex was added to the regression model. Self-reported typical listening volume and Sex accounted for $28.4 \%$ of the variability associated with chosen listening level $(Y=50.6+8.6[$ Loud $]+3.9[$ Sex $])$.

The linear regression model for second survey item asking about being able to hear others when using a PM accounted for only $19.3 \%$ of the variability associated 


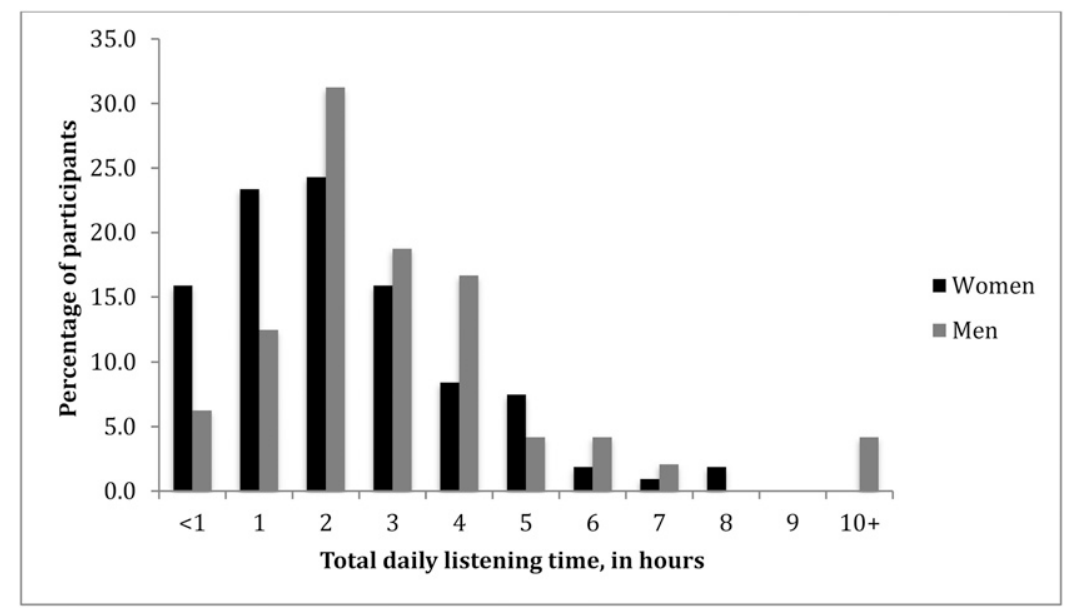

Figure 1. Bar graphs for the reported total daily listening time, in hours, by the 155 participants. Percentage of women is displayed using black bars whereas percentage of men is displayed in gray bars.

with chosen listening level $(Y=58.5+6.2[$ Hear $]$; see "Methods" section for coding of responses to this question). Sex was again added to this model and the item response and Sex accounted for $22.8 \%$ of the variability associated with chosen listening level $(Y=57.9+5.8$ $[$ Hear $]+4.6[\mathrm{Sex}])$.

\section{DISCUSSION}

$\mathrm{T}$ o date, this is the first study in young adults with normal hearing to evaluate the use of survey questions regarding preferred listening volume compared with objectively measured chosen listening level in quiet. Overall mean $L_{\mathrm{DFeq}}$ was $72.5 \mathrm{dBA}$, but young adult men had a significantly higher mean $L_{\mathrm{DFeq}}$ than women; however, 18 of 155 participants did listen at a level $>85$ dBA. Recall, that the FFE conversion used in the present study only accounted for earbud earphones, so if participants used other types of earphones, the chosen listening level will likely be overestimated. Of these 18 participants, 16 (88.9\%) did report that they listened either loud or very loud to the question regarding the most common volume used during a typical day. Furthermore, 15 of the 18 participants (83.3\%) also reported that they have a lot of trouble or cannot hear people when they are listening to a PAS with earphones. The results of the present study demonstrate at least two points. First, approximately $11 \%$ of the participants listen to a PAS with earphones where $>85 \mathrm{dBA}$, but in a quiet background. Second, those who do listen at this level are fairly accurate at reporting this potentially risky behavior.

For the question asking about being able to hear others while using a PAS, specificity was similar to

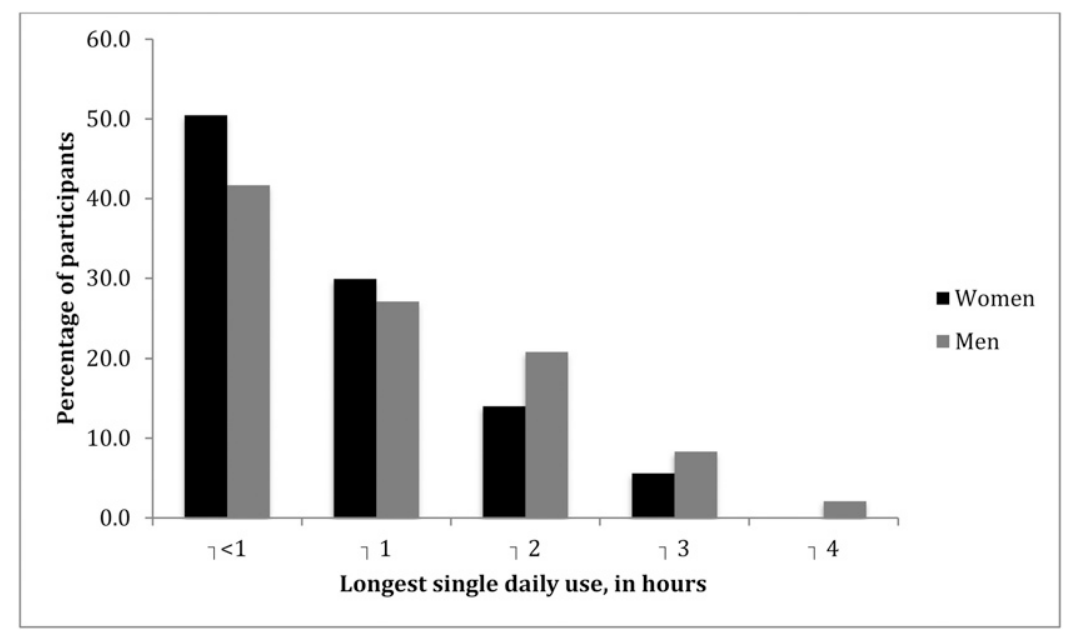

Figure 2. Bar graphs for the reported longest single daily use, in hours, for the 155 participants. Percentage of women is displayed using black bars whereas percentage of men is displayed in gray bars. 


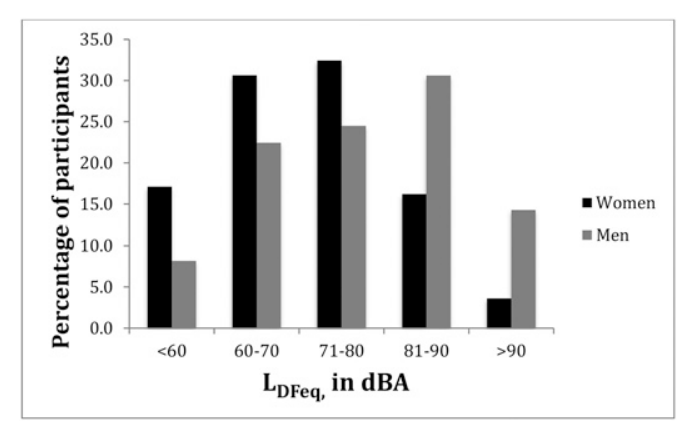

Figure 3. Bar graphs for the measured $L_{\mathrm{DFeq}}$, in dBA, for the 155 participants. Percentage of women is displayed using black bars whereas percentage of men is displayed in gray bars.

sensitivity, but for the question asking about typical volume used, specificity was just more than $70 \%$. Given this result, slightly less than $30 \%$ of participants in the present study reported loud or very loud volume to the typical listening level question, but did not listen at a level $>85 \mathrm{dBA}$. Although $70 \%$ specificity is not a poor result, it is possible that the participants interpreted the question in their everyday environment; in other words, in a background with noise, such as at the gym or walking around campus, rather than in quiet background, like that of a sound-treated room.

Mean $L_{\mathrm{DFeq}}$ of the present study is very similar to what Park et al (2017) reported for the library setting (mean = 70.4-dBA FFE). Park et al (2017) also reported a significant sex difference such that men had a higher chosen listening level, in dBA FFE, in the library environment than women. The sex difference in that environment was approximately $3 \mathrm{~dB}$, but in the present study, men had a chosen listening level that was approximately $6 \mathrm{~dB}$ higher than women. In both studies, participants were young adults enrolled at a university; there are, however, minor differences between the studies. In the present study, 160 (111 women and 49 men) completed the study whereas in Park et al (2017), only 52 university students (15 women and 37 men) had cho-

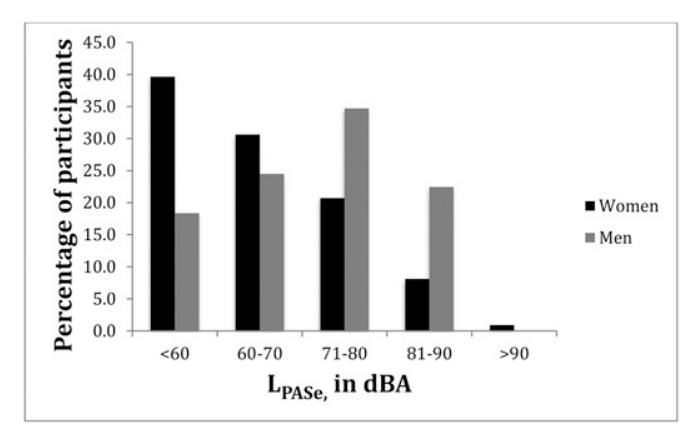

Figure 4. Bar graphs for the calculated $L_{\mathrm{PASe}}$, in dBA, for the 155 participants. Percentage of women is displayed using black bars whereas percentage of men is displayed in gray bars. sen listening levels measured after they were recruited when exiting the library. Although both studies estimated listening risk by compensating for ear canal characteristics and by converting the frequency response of the music to either free-field or diffuse-field, in the present study a probe microphone in the ear canal was used to record the acoustic energy averaged over 1 hour. In contrast, Park et al (2017) measured the level from the PAS using a Jolene mannequin then converted to FFE to estimate risk. The participants in the present study were allowed to change tracks of music and adjust the volume accordingly over the 1 hour in an effort to represent a real-world listening situation. Young adults recruited by Park et al (2017) were instructed to leave the volume and music track set at the point in which they agreed to participate. Some of the participants reported that they had changed the volume when they were approached by researchers to talk with them; it is unclear, however, how many of these participants were from the library condition.

There are substantial similarities between Worthington et al (2009) and the present study. Young adults in both studies were university students and were confirmed to have normal middle ear function and normal hearing sensitivity using tympanometry and pure-tone thresholds, respectively. Both studies used a diffusefield conversion approach using probe microphone measures in the ear canal of participants. Mean $L_{\mathrm{DFeq}}$ in quiet for both studies was approximately $70 \mathrm{dBA}$, although Worthington et al (2009) calculated $L_{\mathrm{DFeq}}$ from a 3-min music sample from 23 participants. Mean $L_{\text {PASe }}$ was slightly higher (66.3 dBA) in the present study than that described by Worthington et al (2009) who reported approximately $60 \mathrm{dBA}$. Based on these daily music exposure calculations for both studies, none of the participants in Worthington et al (2009) listened $>85 \mathrm{dBA}$, but nine participants in the present study had an $L_{\mathrm{PASe}}>85 \mathrm{dBA}$.

This is the first study where performance of two survey questions was examined in an effort to predict listening levels while using a PAS with earphones. As a result, specific test performance (i.e., sensitivity and specificity) of the present study cannot be compared with a previous literature. These results can, however, be compared with other studies on hearing-related test performance of case history questions. Sensitivity values for both questions in the present study were higher than those in the earlier research in older adults and test performance of questions used to predict hearing loss (Clark et al, 1991; Voeks et al, 1993; Nondahl et al, 1998). Compared with other research, specificity values varied depending on the question being evaluated. Specificity in the present study was similar to Nondahl et al (1998) for one question (i.e., typical volume used) but higher than Nondahl et al (1998) for the second question (i.e., hear people). Specificity values 
for both questions were lower than the specificity reported by Clark et al (1991). These studies, however, were primarily in older adults. There are no data in young adults on whether a question can correctly identify hearing loss; this is not surprising given the lower prevalence of hearing loss in younger adults compared with older adults. Because the purpose of this study was to evaluate questions in an effort to identify a potential risk behavior, in this case higher listening levels of music with earphones, higher sensitivity with the potential consequence of lower specificity, is preferred.

In a recent, well-designed study by Johnson et al (2017), a subset of participants completed an 11-item questionnaire to estimate their annual noise exposure (ANE) in both occupational and nonoccupational settings. One aim of this study was to develop a 1-min noise-screening questionnaire to accurately predict ANE. In fact, these researchers used three questions, firearm usage, noisy job, and any other loud noise as the screening tool. Responses to these questions were never $=0$; every few months $=1$; monthly $=2$; weekly $=$ 3 ; and daily $=4$, and a cumulative score $(0-12)$ was calculated. This score was then evaluated for its accuracy in identifying high-risk ANE. A screening score of $\geq 5$ yielded a sensitivity value of $91.7 \%$ and a specificity value of $83.0 \%$; a score that achieved the most balanced values for sensitivity and specificity. The combination of results from Johnson et al (2017) and the present study is important in that there are questions that can be used to identify potentially higher listening levels in young adults from a more transient perspective and questions that be used to identify more cumulative risk exposure.

There are some limitations of the present study. First, as mentioned previously, the conversion used to determine FFE only accounted for earbuds, so it is likely that some measured chosen listening level were overestimated. Second, although the participants were asked to set the volume of their PAS to their chosen listening level, the setting was in a quiet background. This quiet background was a sound-treated room within an auditory research laboratory space, probably the most unlikely place to listen to music for an hour. During the 1 hour, participants were allowed to change musical tracks or adjust the volume accordingly; this was done in an effort to replicate a real-world listening condition. In most cases of daily listening, however, there is some type of background noise present, especially on a university campus. Young adults will turn the level up in the presence of background noise (Worthington et al, 2009; Park et al, 2017) and, depending of the type of earphone used, that noise might also leak into the ear canal creating an additional acoustic source. Second, daily PAS use exposure $\left(L_{\mathrm{PASe}}\right)$ was calculated by combining preferred listening level and the response to a question about typical daily listening time. This exposure will vary considerably, especially for university students. As mentioned previously, background environments will change affecting volume used and daily listening times will vary from day-to-day based on how much free time a university student has. Academic, work, and social schedules are variable, and as a result, PAS use will vary along with them. Last, every effort was made to recruit a somewhat even distribution between women and men, but for this study, there were twice as many women as men. This is not an ideal sex distribution; however, it was representative of the sex distribution in courses offered within the College of Health and Human Services at SDSU from which participants were sampled.

\section{CONCLUSIONS}

$T$ he data from the present study suggest that two very straightforward closed-set questions can be used, either in isolation or included in a clinic's case history form, to accurately identify young adults who might be listening to a PAS with earphones at a high level. In fact, the participants in this study were young adults with normal hearing, and although there is research on the effects of preferred listening levels on short-term changes in auditory function (i.e., distortion product otoacoustic emissions) (Torre and Grace, 2014), the long-term effects of these listening levels is not known. However, if a young adult is identified as indicating this risk behavior, then it is hoped that the behavior can subsequently be modified so as to maintain normal hearing sensitivity as long as possible.

Acknowledgments. The authors would like to thank the San Diego State University students who volunteered for this project and Rachael Cook, Mallery Eppler, Amanda Kaae, Carlee Michaelson, and Shannon O'Donnell for their involvement in data collection.

\section{REFERENCES}

Clark K, Sowers M, Wallace RB, Anderson C. (1991) The accuracy of self-reported hearing loss in women aged 60-85 years. Am J Epidemiol 134(7):704-708.

Danhauer JL, Johnson CE, Byrd A, DeGood L, Meuel C, Pecile A, Koch LL. (2009) Survey of college students on iPod use and hearing health. J Am Acad Audiol 20(1):5-27, quiz 83-84.

Dirks DD, Kincaid GE. (1987) Basic acoustic considerations of ear canal probe measurements. Ear Hear 8(5, Suppl):60S-67S.

Epstein M, Marozeau J, Cleveland S. (2010) Listening habits of iPod users. J Speech Lang Hear Res 53(6):1472-1477.

Hodgetts WE, Rieger JM, Szarko RA. (2007) The effects of listening environment and earphone style on preferred listening levels of normal hearing adults using an MP3 player. Ear Hear 28(3): 290-297.

Hoover A, Krishnamurti S. (2010) Survey of college students' MP3 listening: habits, safety issues, attitudes, and education. Am J Audiol 19(1):73-83. 
Johnson TA, Cooper S, Stamper GC, Chertoff M. (2017) Noise exposure questionnaire: a tool for quantifying annual noise exposure. J Am Acad Audiol 28(1):14-35.

National Institute for Occupational Safety and Health (NIOSH). (1998) Criteria for a Recommended Standard: Occupational Noise Exposure (Revised Criteria 1998). No. 98-126. Cincinnati, OH: US Department of Health and Human Services (NIOSH).

Nondahl DM, Cruickshanks KJ, Wiley TL, Tweed TS, Klein R, Klein BEK. (1998) Accuracy of self-reported hearing loss. Audiology 37(5):295-301.

Occupational Safety \& Health Administration (OSHA). (2007) Occupational Noise Exposure Standard (29 CFR 191.25). Code of Federal Regulations. Washington, DC: US Department of Labor.

Park Y, Guercio D, Ledon V, Le Prell CG. (2017) Variation in music player listening level as a function of campus location. J Am Acad Audiol 28(4):295-313.
Torre P, 3rd. (2008) Young adults' use and output level settings of personal music systems. Ear Hear 29(5):791-799.

Torre P, 3rd, Grace J. (2014) Changes in distortion product otoacoustic emission components after music exposure. J Am Acad Audiol 25(9):804-813.

Torre P, 3rd, Grace J, Hansen C, Millman P, Martin H. (2013) Gender, music, and distortion product otoacoustic emission components. Ear Hear 34(6):e74-e81.

Voeks SK, Gallagher CM, Langer EH, Drinka PJ. (1993) Selfreported hearing difficulty and audiometric thresholds in nursing home residents. J Fam Pract 36(1):54-58.

Worthington DA, Siegel JH, Wilber LA, Faber BM, Dunckley KT, Garstecki DC, Dhar S. (2009) Comparing two methods to measure preferred listening levels of personal listening devices. J Acoust Soc Am 125(6):3733-3741. 